\title{
Temperamento, comportamento e experiência dolorosa na trajetória de desenvolvimento da criança
}

\author{
Vivian Caroline Klein \\ Maria Beatriz Martins Linhares \\ Universidade de São Paulo, Ribeirão Preto, Brasil
}

\begin{abstract}
Resumo: O presente estudo teve por objetivo realizar uma revisão de estudos sobre as relações entre temperamento, comportamento e experiências dolorosas iniciais nas trajetórias de desenvolvimento de crianças, especialmente nas nascidas prematuras. Os estudos empíricos foram obtidos por meio das bases de dados PsycINFO e Medline. Os resultados mostraram que o temperamento foi preditor de padrões individuais de reação à dor e problemas de comportamento da criança, em diferentes idades. Além disso, as experiências precoces de dor em crianças nascidas prematuras podem afetar a reatividade do bebê, que por sua vez mostrou associação com dimensões do temperamento nos primeiros anos de vida.
\end{abstract}

Palavras-chave: Temperamento. Reatividade. Dor. Prematuridade.

\section{Temperament, behavior and painful experience in the child developmental course}

\begin{abstract}
The objective of the present study was to review the studies about the relations among the temperament, behavior, and early painful experiences in children developmental pathways, especially with preterm born children. The empirical studies were obtained through the data bases PsycINFO and Medline. The results showed that the temperament was a predictor of the individual patterns of reaction to pain and to behavior problems at different ages. Moreover, the early painful experiences of children born preterm can influence infant's reactivity, which was in association to temperamental dimensions during the first years of life.
\end{abstract}

Keywords: Temperament. Reactivity. Pain. Prematurity.

\section{Temperamento, comportamiento y experiencia dolorosa en la trayectoria del desarrollo del niño}

\begin{abstract}
Resumen: El objetivo del presente estudio fue realizar una revisión sobre las relaciones entre temperamento, comportamiento y experiencias dolorosas iniciales en la trayectoria del desarrollo de los niños, especialmente en los nacidos prematuros. Los estudios empíricos fueron obtenidos por medio de la base de datos PsycINFO y Medline. Los resultados muestran que el temperamento fue el definidor de los patrones individuales de las reacciones al dolor y problemas del comportamiento de los niños, en diferentes edades. También las experiencias precoces de dolor en niños nacidos prematuros pueden afectar la reactividad del recién nacido, que además mostró asociación con dimensiones del temperamento en los primeros años de vida.
\end{abstract}

Palabras clave: Temperamento. Reactividad. Dolor. Prematuridad. 
A Psicopatologia do Desenvolvimento, há mais de duas décadas, tem recebido crescente reconhecimento no estudo sobre os transtornos de desenvolvimento à luz do contexto do psicológico normativo (Luthar, Burack, Cicchetti \& Weisz, 1997). Ela incorpora os parâmetros do desenvolvimento à compreensão das psicopatologias, entendendo as desordens de adaptação do indivíduo sob a ótica do processo e do contexto, sem predições lineares, deterministas ou isomórficas (Rutter \& Sroufe, 2000). Investiga a natureza do processo de desenvolvimento, as origens e os cursos de padrões individuais de adaptação comportamental, assim como os fatores e mecanismos que influenciam positiva ou negativamente estas diferentes trajetórias de vida (Achenbach, 1992; Garber, 1984; Sameroff, 2000).

Esta perspectiva preconiza os intercâmbios probabilísticos entre dinâmicas individuais e de contextos ambientais que compreendem o comportamento humano, em oposição a relações lineares de causaefeito. Desta forma, verifica-se um avanço em comparação aos modelos tradicionais de compreensão da doença mental, ao incorporar funcionamentos biológicos e comportamentais na perspectiva de sistemas de regulação desenvolvimental (Sameroff, 2000). Além disso, ela integra o conhecimento inter- e intradisciplinar, teorias, contextos e domínios de pesquisa, transcendendo de um nível descritivo de sintomas isolados para um de compreensão de processos presentes em trajetórias de desenvolvimento típico ou atípico (Cicchetti \& Cohen, 1995).

Dada a importância do estudo de múltiplas influências, alguns conceitos nesta perspectiva são fundamentais para a compreensão das trajetórias de desenvolvimento das crianças. O primeiro é o de fator de risco, que é uma variável positivamente relacionada a um resultado desenvolvimental negativo (Gutman, Sameroff \& Cole, 2003) e que quando presente, aumenta a probabilidade de o indivíduo apresentar problemas físicos, psicológicos ou sociais (Yunes \& Szymanski, 2001). Os fatores de risco podem ser identificados na pessoa ou no ambiente e de forma combinada em ambos (Kopp \& Kaler, 1989), e devem ser entendidos como um indicador potencial que aumenta a probabilidade da ocorrência de efeitos negativos no desenvolvimento, não sendo considerado uma variável fixa e pontual. Nesse sentido a análise dos fatores de risco precisa contemplar a multiplicidade dos concomitantes e os seus mecanismos de ação.

O segundo conceito é o de fator de promoção, que é uma variável positivamente relacionada a um resultado desenvolvimental positivo, independente da exposição do indivíduo a riscos (Gutman \& cols., 2003).

$\mathrm{O}$ terceiro conceito é o de mecanismo de proteção, que consiste em uma variável positivamente relacionada a um resultado desenvolvimental positivo em um grupo de alto risco, porém não num de baixo risco, apresentando efeito interativo (Gutman \& cols., 2003). Os mecanismos de proteção podem ser atributos disposicionais do indivíduo, aspectos do meio ambiente ou da interação entre esses, que modificam, melhoraram ou alteraram respostas pessoais a determinados riscos de desadaptação (Rutter, 1987; Werner, 1986); desta forma, neutralizando os efeitos adversos provocados por uma condição de risco.

O quarto conceito é o de vulnerabilidade, uma variável negativamente relacionada a um resultado desenvolvimental positivo em um grupo de baixo risco, porém não em um de alto risco (Gutman \& cols., 2003).

O quinto conceito é o de resiliência, que se refere a processos que explicam a superação de crises e adversidades em indivíduos, grupos ou organizações (Rutter, 1987; Yunes \& Szymanski, 2001; Yunes, 2003). A resiliência envolve a interação entre atributos e características do indivíduo e recursos ambientais em um processo que resulta em superação e retorno ao padrão adaptativo inicial, sem que o indivíduo saia ileso deste processo.

Neste interjogo entre risco e proteção se interpõem variáveis moderadoras e mediadoras que ajudam a compreender o efeito das diferentes forças que desencadeiam os resultados desenvolvimentais. A moderadora é uma variável que especifica uma condição sob a qual um dado preditor é relacionado a um resultado desenvolvimental. A natureza da associação entre preditor e predito pode variar em função do moderador. Por outro lado, a mediadora é uma variável que explica o processo ou mecanismo pelo qual um preditor significativamente afeta um resultado desenvolvimental; este liga-se ao mediador, o qual, por sua vez, o é à variável predita (Holmbeck, 2002). 
Nesse contexto, o presente estudo tem por objetivo contribuir para a compreensão dos processos envolvidos na trajetória de desenvolvimento da criança, com enfoque na interação entre variáveis individuais dela e as ambientais que produzem resultados desenvolvimentais. Inclui a análise de estudos empíricos, obtidos por meio das bases de dados PsycINFO e Medline, que tratam das relações entre temperamento, comportamento e experiências de dor em crianças nascidas a termo e a pré-termo.

Portanto, tem-se primeiro uma breve descrição das abordagens teóricas que norteiam as investigações do temperamento, com ênfase na abordagem inserida na perspectiva desenvolvimental proposta por Rothbart (1981). Segundo, os resultados de estudos empíricos que relacionam temperamento, reatividade e comportamento em crianças nascidas a termo. Terceiro, os resultados de estudos empíricos que relacionam temperamento, comportamento e reatividade à dor em crianças nascidas prematuras. Por fim, os de estudos que demonstram os efeitos a curto e médio prazo destas experiências cumulativas de dor na reatividade da criança nascida prematura.

A condição de nascimento prematuro consiste em um fator de risco biológico ao desenvolvimento da criança, tornando-a vulnerável (Werner, 1986; Laucht, Esser \& Schmidt, 2001, 2002), exposta a experiências precoces cumulativas de dor na Unidade de Terapia Intensiva Neonatal e com maior propensão a apresentar problemas de desenvolvimento, em especial, de comportamento em sua trajetória de vida (Grunau, Whitfield \& Petrie, 1994).

\section{Relevância do estudo do temperamento para a compreensão do interjogo entre risco e prote- ção na trajetória de desenvolvimento da criança}

Diferentes crianças podem responder aos mesmos investimentos sociais de promoção do desenvolvimento de formas diferentes, com suas características individuais influenciando trajetórias tanto de sucesso quanto de desadaptação, e podem também determinar se uma intervenção é necessária, assim como as estratégias que o adulto deve escolher para promover mudanças. Entre essas características pessoais relevantes destaca-se o temperamento, e a pesquisa nesta área permite estudar interações entre influências individuais e ambientais, pois descreve pro- cessos evidentes precocemente na vida a partir dos quais as adaptações sociais às condições ambientais se desenvolvem.

No campo de estudo do temperamento, uma primeira abordagem adveio de um trabalho pioneiro realizado por Thomas e Chess denominado Estudo Longitudinal de Nova Iorque (Thomas, Chess, Birch, Hertzig \& Korn, 1963 citado por Muris \& Ollendick, 2005). Nela, o temperamento é entendido como uma categoria derivada da constelação de comportamentos exibidos em um determinado momento de vida, que são resultantes de todas as influências passadas e presentes, as quais os modelam e modificam em um processo constantemente interativo. A consistência de traços ou constelações de temperamento em um indivíduo ao longo do tempo, no entanto, deve requerer estabilidade nestas forças interacionais, tais como influências ambientais, motivações e habilidades.

Foi desenvolvido um sistema de nove categorias principais para classificar o temperamento, a saber: 1) nível de atividade, referente ao componente motor presente no funcionamento de uma criança $\mathrm{e}$ na proporção diurna de períodos de ativação e passividade; 2) ritmo, direcionado à previsibilidade e/ ou imprevisibilidade no tempo de qualquer função; 3 ) aproximação ou retraimento, ligado à resposta inicial a um estímulo novo, por exemplo, uma nova comida ou um novo brinquedo; 4) adaptabilidade, que se refere à facilidade com que a criança modifica uma situação nova ou alterada na direção desejada; 5) limiar de responsividade, voltado à intensidade do nível de estimulação necessária para evocar uma resposta deliberada, independente da forma específica que esta pode assumir ou da modalidade sensorial afetada; 6) intensidade de reação, que diz respeito ao nível de energia da resposta, independentemente da sua qualidade ou direção; 7) qualidade de humor, que fala da quantidade de prazer, divertimento ou comportamento amistoso em comparação ao desprazer, choro e comportamento não amistoso; 8) distraibilidade, direcionada à efetividade de um estímulo ambiental externo em interferir no comportamento vigente e alterar sua direção; 9) período de atenção e persistência, referente respectivamente ao período de tempo que uma atividade particular é realizada pela criança e a continuação de uma na pre- 
sença de obstáculos para a manutenção da direção da atividade (Muris \& Ollendick, 2005).

Segundo Rothbart, Ellis, Rueda e Posner (2003), devido ao fato de estas dimensões terem sido desenvolvidas com objetivos clínicos, não houve uma tentativa inicial de elaborar medidas homogêneas e relativamente independentes baseadas nestes constructos. Desta forma, questionários e outros instrumentos que se fundamentam nesta abordagem lidam com constructos que muitas vezes apresentam sobreposição de significados.

A partir dessas dimensões, foram obtidas categorias sobre tipos de temperamento, a saber: fácil, difícil, intermediário e lento. Há na literatura o uso extensivo do constructo criança difícil. Porém, Rothbart (2004) destaca vários problemas relacionados a sua utilização na área da pesquisa, como a ampla variação nas suas operacionalizações, a confusão dos conceitos de criança difícil, com problema de comportamento e os problemas de adaptação social advindos do rótulo a ela dado.

Em uma segunda abordagem de temperamento, denominada Modelo EAS - Emocionalidade, Atividade e Sociabilidade (Buss \& Plomin, 1975 citado por Muris \& Ollendick, 2005) distinguiramse três dimensões básicas de temperamento, a saber: a) emocionalidade, que se refere à instabilidade psicológica e à propensão a experimentar sentimentos de medo, raiva e tristeza; b) atividade, que diz respeito a características como tempo, vigor e resistência, podendo ser medida por taxa e amplitude da fala e movimento, deslocamentos corporais e duração de comportamento agitado; c) sociabilidade, direcionada à tendência de afiliação e responsividade às pessoas, podendo ser avaliada pela preferência por estar com os outros, necessidade de compartilhar atividades e de receber atenção como resultado da interação social.

As abordagens de Thomas e Chess (1977) Buss e Plomin (1984) assumem que as diferenças individuais do temperamento refletem estilos de resposta comportamental e que os processos temperamentais são equivalentes aos afetivos, sendo consideradas como estilísticas do temperamento, pois se preocupam em descrever como os comportamentos ocorrem em um determinado tipo de temperamento e não no porquê dele (motivação) ou $o$ quê (ação) (Helgadóttir \& Wilson, 2004).

Em uma terceira abordagem, proposta por Rothbart (1981), o temperamento é composto por dimensões relacionadas ao modelo da Psicopatologia do Desenvolvimento. Segundo Goldsmith (1983), a teoria e a pesquisa de Rothbart mudaram a concepção anterior de temperamento de uma definição estilística para uma psicobiológica. Portanto, ele não seria restrito aos processos afetivos e incluiria as diferenças individuais na auto-regulação da atenção como sua dimensão básica. Nesta abordagem o funcionamento do temperamento é visto como influenciado tanto pela maturação quanto pela experiência (Rothbart, Posner \& Hershey, 1995). Este último aspecto constitui-se em uma contribuição fundamental para a compreensão dele na perspectiva desenvolvimental.

A Abordagem de Rothbart (2004) define temperamento como diferenças individuais com base constitucional na reatividade e auto-regulação, observadas nos domínios de emocionalidade, atividade motora e atenção, sendo influenciados ao longo do tempo pela hereditariedade, maturação e experiência. A reatividade é definida como característica da responsividade individual a mudanças de estimulação apresentada em diversos níveis (comportamental, autonômico, neuro-endócrino) e por meio de parâmetros de latência, tempo de aumento, de recuperação da reação e intensidade máxima. A autoregulação consiste em processos que modulam essa reatividade, incluindo aproximação/ retraimento comportamental, controle inibitório e de atenção. Com o curso do desenvolvimento da criança, sistemas inicialmente mais reativos tornam-se crescentemente regulados, na medida em que os de inibição ao medo e de controle de atenção se desenvolvem.

Apesar de o temperamento pertencer a disposições relativamente estáveis, seus eliciadores e expressões freqüentemente mudam ao longo do curso desenvolvimental (Putnam, Ellis \& Rothbart, 2001; Rothbart \& Putnam, 2002; Rutter, 1994). A princípio, os comportamentos iniciais podem ser vistos como reativos a eventos de estimulação imediata ou a mudanças endógenas. Posteriormente, no entanto, sistemas de regulação mais diretos, incluindo aspectos de inibição de medo e de flexibilidade de controle de 
atenção, irão se desenvolver para modular essa reatividade comportamental (Rothbart \& Putnam, 2002). Na medida em que o desenvolvimento envolve continuidades e descontinuidades certa consistência é esperada, podendo adotar diferentes formas ao longo do tempo (Rutter, 1994).

Segundo Rothbart e Putnam (2002), as diferenças individuais do temperamento constituem a expressão mais precoce da personalidade e o substrato a partir do qual ela se desenvolve. Enquanto a personalidade da criança incluirá habilidades, hábitos e estruturas cognitivas moldadas por meio da interação com o ambiente, o temperamento provê as bases biológicas sobre as quais estas estruturas são construídas. Ao traçar o desenvolvimento do temperamento e as adaptações que ele sustenta em diferentes momentos do ciclo vital, torna-se possível avançar na compreensão do risco para psicopatologias.

O temperamento pode moldar e ser moldado pela adaptação individual por meio de diferentes trajetórias (Rothbart, Posner \& Hershey, 1995), ocorrendo de formas diversas, nas quais ele estaria envolvido no desenvolvimento do risco para psicopatologias, incluindo: a) diferenças individuais, que em seu extremo, que se constituiriam em psicopatologia ou predisporiam o indivíduo à mesma; b) características de temperamento que evocam reações em outras pessoas, podendo promover ou neutralizar o risco para psicopatologia; c) ou influenciar a seleção dos nichos de desenvolvimento da pessoa expondo-a a maior ou menor risco para psicopatologia; d) o temperamento podendo influir na modalidade de um transtorno, no seu curso e na probabilidade de sua recidiva; ou no processamento de informação sobre si mesmo e o mundo, seja aumentando ou diminuindo a probabilidade para psicopatologia; e) ainda regular ou neutralizar os fatores de risco ou estresse; f) há variação na responsividade temperamental aos eventos ambientais; g) na interação entre os sistemas, sendo que alguns deles se desenvolvem tardiamente; $h$ ) as disposições temperamentais podem moldar diferentes trajetórias para um dado resultado desenvolvimental e as individuais levar a múltiplos resultados; i) as características dele e do ambiente de cuidado trazer contribuições independentes ao desenvolvimento, ou interagir aumentando ou diminuindo o risco para um transtorno; j) os transtornos por si próprios podem mudar aspectos do temperamento e ocasionar riscos futuros.

Temperamento, comportamento e reatividade da criança

A relação entre temperamento e problemas de comportamento da criança tem sido estabelecida em diferentes idades (Rothbart \& cols., 1995). No estudo de Wasserman, Diblasio, Bond, Young e Colletti (1990) foi encontrado que o temperamento difícil da criança aos quatro meses, assim como o baixo nível sócio-econômico, predisse problemas de comportamento aos seis anos de idade em uma amostra clínica pediátrica. Segundo Bates, Pettit, Dodge e Ridge (1998), as crianças com temperamento resistente (difícil de conduzir e com falta de auto-regulação) mostraram níveis mais altos de problemas de externalização na idade escolar.

A reatividade tem sido incluída como um dos traços centrais do temperamento (Rothbart, 1989; Strelau \& Eliaz, 1994). A influência dele na reatividade da criança aos três anos de idade foi investigada por Zimmerman e Stansburry (2004) e avaliada por meio da variação do nível de cortisol salivar medido antes, durante e depois de uma situação experimental em que a criança é abordada por pessoa estranha. Os resultados mostraram que alto nível de timidez dela (velocidade de aproximação baixa ou inibida e desconforto em situações sociais) foi preditor de elevação de um relevante parâmetro fisiológico de estresse (nível de cortisol salivar), enquanto que boa regulação emocional e freqüentar creche foram preditores de baixas elevações nos níveis de cortisol.

A relação entre nível de cortisol salivar em uma situação estressante, apego, temperamento e desenvolvimento de crianças aos 15 meses de idade foi examinada por Van Bakel e Riksen-Walraven (2004). Os autores identificaram que as que apresentaram altos níveis de propensão à raiva (a reações negativas particularmente em situações de frustração) e melhor desempenho cognitivo apresentaram maior elevação do nível de cortisol salivar. Houve correlação positiva entre desenvolvimento cognitivo e aumento de cortisol salivar apenas no grupo de bebês com baixos escores de apego seguro. Desta forma, este foi identificado como variável moderadora da relação entre desenvolvimento cognitivo e reatividade fisiológica da criança ao estresse. 
A relação entre temperamento do bebê nascido a termo no primeiro mês de vida, e choro e inquietação, avaliados ao longo de 16 dias consecutivos aos dois meses de vida, foi objeto do estudo de Blum, Taubman, Tretina e Heyward (2002). Os resultados mostraram o maior tempo de choro e inquietação associado com bebês de temperamento com alta intensidade de resposta e baixa distraibilidade. Estes resultados sugerem que bebês com altos níveis de choro são mais reativos à estimulação sensorial e difíceis de consolar do que os que choram menos. Certamente, o modo como o ambiente vai lidar com essas características temperamentais deles irá contribuir para o delineamento de trajetórias de desenvolvimento. A interação entre a criança e seus cuidadores precisará ser regulada para lidar com as características pessoais à luz do contexto de influência bidirecional entre indivíduos.

A relação entre o temperamento e a reatividade à dor têm sido destacada no campo de investigação devido ao fato de este tipo de reatividade ser considerado uma das dimensões da reatividade ao estresse e da capacidade de auto-regulação do indivíduo (Morison \& cols., 2003). O estudo de Helgadóttir e Wilson (2004) examinou as relações entre temperamento e o auto e hetero-relato de dor em crianças na faixa de três a sete anos durante o procedimento de tonsilectomia; os resultados revelaram que maior intensidade de dor pós-operatória durante a hospitalização foi relatada pelas mais ativas ou com humor negativo; no pós-alta, maior intensidade de dor pós-operatória pelas que apresentaram maior distraibilidade ou menor limiar sensorial.

$\mathrm{Na}$ busca de identificar preditores de somatização e reatividade à dor durante procedimento doloroso aos cinco anos de idade, Rocha, Prkachin, Beaumont, Hardy e Zumbo (2003) avaliaram o temperamento da criança nascida a termo, a quantidade de experiências prévias de dor dela e dos modelos de dor (pessoas de seu convívio portadores de dor crônica), a resposta materna durante o procedimento, $o$ encorajamento parental do comportamento de doença da criança. Os autores encontraram que temperamento caracterizado como de baixa adaptabilidade, bem como maior exposição prévia a experiências dolorosas, foi preditor de maior reatividade à dor da criança observada durante procedimento doloroso, que, por sua vez, predisse o relato parental de somatização da criança.

A realização de futuras pesquisas sobre o papel do temperamento e das experiências de exposição a estímulos dolorosos no desenvolvimento de estratégias para lidar com as dolorosas e de somatização da criança parece ser um caminho promissor para a melhor compreensão das relações entre temperamento e reatividade à dor. Essa linha de investigação se faz necessária, principalmente, para estudar os desfechos de trajetórias de desenvolvimento em populações biologicamente vulneráveis constituídas por crianças de alto risco para problemas na área de saúde mental.

\section{Comportamento e temperamento da criança nascida prematura exposta precocemente a ex- periências inevitáveis de dor}

Uma amostra vulnerável que freqüentemente se encontra exposta a experiências inevitáveis de dor é constituída por bebês prematuros, considerados em situação de risco biológico que ameaça o seu desenvolvimento (Werner, 1986), constituindo-se em risco em si mesmo, além de expô-los a uma cadeia de adversidades decorrentes da própria prematuridade (Beckwith \& Rodning, 1991; Laucht, Esser \& Schimdt, 1997; Linhares, Bordin \& Carvalho, 2004), dentre as quais encontra-se a alta exposição do bebê a estímulos dolorosos inerente aos procedimentos médicos e de enfermagem necessários ao cuidado intensivo do bebê no período de internação na Unidade de Terapia Intensiva Neonatal (UTIN).

Os resultados desenvolvimentais do grupo de crianças nascidas pré-termo com peso ao nascimento abaixo de 1500 gramas mostraram que estas, quando comparadas às nascidas a termo, apresentam mais deficiência cognitiva (Bradley \& cols., 1993; Damman \& cols., 1996; Laucht \& cols., 1997, 2002), problemas de desempenho escolar (Taylor, Hack, Klein \& Schatschneider, 1995; Withfield \& Grunau, 2000), dificuldades comportamentais (Laucht \& cols., 2002) e somatização (Grunau, Whitfield \& Petrie, 1994).

Diversos estudos têm identificado o comportamento como um dos principais problemas na trajetória de desenvolvimento de crianças nascidas com o risco biológico da prematuridade. Schothorst e Van Engeland (1996) encontraram que as pré-termo com idade gestacional igual ou inferior a 32 semanas e as 
pré-termo e pequenas para a idade gestacional apresentaram mais problemas sociais na idade escolar em comparação às nascidas a termo, sendo que esses problemas persistiram até a adolescência. Resultados semelhantes foram obtidos por Foulder-Hughes e Cooke (2003). No estudo prospectivo longitudinal de Gray, Indurkhya e McCormick (2004) foi identificada a prevalência e continuidade de problemas de comportamentos aos três, cinco e oito anos de idade em 869 crianças nascidas prematuras com peso abaixo de $2500 \mathrm{~g}$. Os autores encontraram uma prevalência de problemas de comportamento nas crianças de $20 \%$ nas idades avaliadas, sendo o dobro da vista na população em geral. Os problemas de comportamento foram mantidos ao longo do tempo nas diferentes idades estudadas em $50 \%$ da amostra.

Considerando os estudos sobre nascimento prematuro e problemas de comportamento da criança, fazse necessário avançar na compreensão a respeito de como as características individuais das nascidas prematuras podem atuar na trajetória de seu desenvolvimento.

A relação entre temperamento e comportamento da criança nascida prematura foi identificada em alguns estudos. Alto escore global de temperamento dificil, de índice de estresse parental e família de pais solteiros foram preditores de problemas de comportamento das prematuras aos sete anos (Saylor, Boyce \& Price, 2003). A alta emocionalidade negativa aos três anos de vida foi encontrada em grupos de crianças prematuras diferenciadas quanto à presença da dimensão externalização e internalização do comportamento, respectivamente, em comparação com as do grupo controle sem problemas de comportamento (Blair, 2002).

Estudos que compararam crianças nascidas prematuras com as a termo em relação ao temperamento encontraram diferenças significativas entre os grupos. Considerando as categorias diagnósticas de temperamento difícil e fácil, dois estudos não encontraram diferenças entre crianças nascidas prematuras e as a termo aos seis (Honjo \& cols., 2002) e vinte e quatro meses (Sajaniemi, Salokorpi \& Von Wendt, 1998). No entanto, ao se considerar as dimensões do temperamento das crianças os estudos encontraram especificidades relacionadas ao grupo das prematuras. No primeiro ano de vida, a comparação das dimensões do temperamento entre os grupos revelou que as crianças nascidas prematuras eram mais retraídas em se aproximar de pessoas ou situações desconhecidas (Honjo \& cols., 2002), mais ativas (Kerestes, 2005) e mais difíceis para serem consoladas (Larroque, N'guyen, The Tich, Guedeney, Marchand \& Burguet, 2005) do que as a termo. Estes achados vão à direção oposta aos de um estudo anterior realizado por Sajaniemi e cols (1998) no segundo ano de vida, o qual identificou que as crianças nascidas prematuras foram menos ativas, mais adaptáveis, menos intensas, com humor mais positivo e com menor limiar de resposta à estimulação, independente do nível de gravidade de estado clínico na fase neonatal.

Algumas variáveis relacionadas à condição de saúde da criança nascida prematura foram identificadas em associação com dimensões do temperamento. Em relação à idade gestacional, quanto menor ela for, menor o período de tempo de atenção no primeiro ano de vida (Kerestes, 2005). Quanto as intercorrências clínicas decorrentes da prematuridade, as prematuras diagnosticadas com lesões cerebrais no período neonatal e com atraso no desenvolvimento psicomotor eram menos responsivas a estímulos sociais e adaptáveis aos 9 meses do que as nascidas na mesma condição que não apresentaram tais problemas (Larroque \& cols., 2005). Apenas um estudo encontrou associação entre maior morbidade perinatal à maior persistência de atenção durante as atividades (Weiss, St. John-Seed \& Wilson, 2004), o que surpreendeu os autores. No entanto, estes supõem que as complicações médicas durante o período neonatal podem prenunciar certos problemas físicos ou neurológicos que limitam as habilidades da criança e resultam em mais dificuldades para terminar uma tarefa. Estas limitações poderiam requerer da criança que focalizasse ou prestasse atenção a fim de efetivamente engajar-se em determinada atividade ou realizar a tarefa.

Grunau, Whitfield e Petrie (1994) encontraram que o temperamento foi significativamente relacionado à sensibilidade à dor no grupo de crianças nascidas a termo, e moderadamente no das pré-termo de baixo peso (1500- $2499 \mathrm{~g}$ ) e de muito baixo peso (801-1499g) e não relacionado à sensibilidade à dor no grupo de extremo baixo peso $(<801 \mathrm{~g})$ aos dois anos de idade. Assim, nas nascidas a termo e nas pré-termo com peso acima de 801 gramas, maior 
reatividade emocional foi relacionada a maior resposta à dor. Desta forma, o temperamento foi um mediador significativo da sensibilidade à dor, porém, identificou-se a relação entre menor peso de nascimento e o papel decrescente do temperamento como mediador de sensibilidade à dor aos dois anos de idade da criança. Estes achados indicam que nas mais imaturas pode ocorrer a atuação de outras variáveis mediadoras da relação entre temperamento e sensibilidade à dor.

Esses achados evidenciam a importância de serem investigadas outras variáveis clínicas do bebê, assim como ambientais, no estudo do temperamento da criança nascida prematura. Nesse sentido, alguns estudos destacam as experiências iniciais de dor como variável ambiental que pode influenciar dimensões de temperamento, mais especificamente relacionada à reatividade do nascido prematuro.

Grunau e cols (2001) verificaram que os bebês nascidos com extremo baixo peso ao serem expostos a procedimento de lancetagem para coleta de sangue aos oito meses de idade apresentaram maiores taxas basais de batimento cardíaco e recuperação comportamental e fisiológica mais rápida do que os a termo. Além disso, entre os com extremo baixo peso ao nascimento, maior número de procedimentos invasivos e dolorosos prévios foram associados a menor atividade facial de resposta à dor e menor taxa de batimentos cardíacos durante o procedimento doloroso.

Outro estudo encontrou níveis significativamente mais altos de cortisol salivar em bebês nascidos com idade gestacional extremamente baixa (d" 28 semanas) aos oito meses de idade cronológica corrigida antes e depois da apresentação de um estímulo visual envolvendo novidade em comparação aos com idade gestacional muito baixa (29-32 semanas) e com os a termo. Após controlar a gravidade neonatal e a quantidade de exposição à oxigenação artificial na UTIN, verificou-se que níveis mais elevados de cortisol basal em bebês pré-termo aos oito meses foram associados com maior número de procedimentos invasivos e dolorosos ocorridos no período neonatal (Grunau, Weinberg \& Whitfield, 2004).

Avançando no estudo da relação entre quantidade de exposição à dor no período neonatal e reatividade, Grunau e cols (2005) encontraram que nos de menor idade gestacional ( $<28$ semanas), maior quantidade de exposição neonatal à dor foi preditora de menor resposta de cortisol plasmático ao estresse e reatividade facial à dor na $32^{\mathrm{a}}$ semana de idade cronológica corrigida, independente da gravidade clínica neonatal e da quantidade de exposição à morfina a partir do nascimento. No grupo de idade gestacional superior a 28 semanas, a maior gravidade do estado de saúde neonatal foi significantemente associada a menor frequiência de batimento cardíaco durante o procedimento doloroso.

Esses estudos mostram que tanto a prematuridade quanto as condições adversas decorrentes da terapia intensiva neonatal, que envolvem procedimentos dolorosos inevitáveis no início do desenvolvimento, constituem-se em mecanismos que afetam a reatividade comportamental e fisiológica das crianças à dor. No entanto, é importante destacar as dimensões e categorias de temperamento, parâmetros comportamentais e fisiológicos de reatividade da criança e características biológicas e ambientais decorrentes do nascimento prematuro.

Sendo assim, os estudos analisados avaliaram também outras variáveis, tais como: a) tipo de estratégias de regulação emocional (Zimmermann \& Stansbury, 2004), desenvolvimento cognitivo e apego (Van Bakel \& Riksen-Walraven, 2004); b) variáveis parentais, como atitudes relacionadas ao uso de analgésicos (Helgadóttir \& Wilson, 2004), resposta materna ao comportamento da criança durante o procedimento doloroso e encorajamento parental ao de doença da criança (Rocha, Prkachin, Beaumont, Hardy \& Zumbo, 2003), estresse e estilo parental (Saylor, Boyce \& Price, 2003), (Grunau, Whitfield \& Petrie, 1994); c) Variáveis familiares, do tipo nível sócio-econômico (Gray, Indurkhya \& McCormick, 2004; Wasserman \& cols., 1990); quantidade de pessoas do convívio da criança que são portadores de dor crônica e servem como modelos (Rocha \& cols., 2003) nível de suporte e tipo de composição familiar (Saylor, Boyce \& Price, 2003).

\section{Considerações Finais}

O temperamento consiste em uma variável individual fundamental para a compreensão das trajetórias de desenvolvimento das crianças. A reatividade é um aspecto considerado em diferentes abordagens 
teóricas sobre o temperamento. Os estudos a avaliam com parâmetros comportamentais (choro, mímica facial) e fisiológicas (cortisol salivar, batimento cardíaco). Altos escores nas dimensões timidez, raiva e intensidade de resposta e baixos em distraibilidade foram relacionados à maior reatividade da criança.

A reatividade à dor envolve aspectos básicos do temperamento. Níveis altos de atividade e humor negativo e baixos de adaptabilidade foram relacionados a maior reatividade à dor da criança. Mais exposição prévia de dor e maior reatividade a ela foram associados à somatização da criança.

O grupo de crianças nascidas prematuras foi destacado no presente artigo considerando que este se encontra muito precocemente e de forma persistente exposto a experiências de dor e é identificado como de alto risco para problemas de comportamento, em idades posteriores. Em crianças prematuras, temperamento difícil e maior emocionalidade negativa foram associados a mais problemas de comportamento.

A comparação do temperamento de prematuras com a termo revelou que as dimensões de temperamento mais do que suas categorias diagnósticas de fácil e difícil foram sensíveis em identificar as especificidades do grupo de crianças nascidas prematuras. Estas foram mais retraídas, ativas e difíceis de consolar do que as a termo.

Consideraram-se não somente o peso de nascimento e a idade gestacional para identificar as características de temperamento de crianças nascidas prematuras. Maior gravidade clínica neonatal das prematuras foi associada a menores escores em persistência de atenção, responsividade social e adaptação. Os estudos que avaliaram a quantidade de exposição prévia à dor na UTIN com a reatividade de crianças nascidas prematuras foram inconsistentes em revelar a direção da associação entre essas variáveis.

Sendo assim, a análise das trajetórias de desenvolvimento das crianças prematuras deve levar em conta as características de gravidade de saúde neonatal, bem como da exposição à estimulação dolorosa na UTIN, considerando que alguns estudos relacionaram aspectos do temperamento a estas variáveis mais do que à condição de nascimento prematuro em si mesma.
Faz-se assim necessário o investimento em pesquisas que investiguem os processos que relacionam características individuais, como temperamento da criança, e ambientais, como história de exposição à estimulação dolorosa em idades precoces, e resultados desenvolvimentais da criança.

Considerando que o grupo de prematuras é biologicamente vulnerável e que as variáveis de sua saúde exercem influência no seu temperamento, estudos que avaliam o temperamento destas crianças por meio do referencial psicobiológico de Rothbart podem identificar especificidades neste grupo relacionadas a auto-regulação e controle de atenção.

A detecção precoce de características individuais que podem influenciar o desenvolvimento da criança, bem como a compreensão acerca do interjogo entre fatores constitucionais e ambientais no processo desenvolvimental, contribuem para a promoção do desenvolvimento da criança, assim como para a prevenção de problemas de saúde mental.

\section{Referências}

Achenbach, T. M. (1992). Developmental psychopathology. In M. H. Bornstein \& M. E. Lamb (Orgs.), Developmental Psychology: An advanced textbook (pp.629-676). New York: Lawrence Erlbaum Associates Publishers.

Bates, J. E., Pettit, G. S., Dodge, K. A., \& Ridge, B. (1998). Interaction of temperamental resistance to control and restrictive parenting in the development of externalizing behavior. Developmental Psychology, 34, 982-95.

Beckwith, L., \& Rodning, C. (1991). Intellectual functioning in children born preterm: Recent research. In L. Okagaki \& R.J. Sternberg (Eds.), Directors of development-influences on the development of children's thinking (pp. 25-28). New Jersey: Lawrence Erlbaum Associates Publishers.

Blair, C. (2002). Early intervention for low birth weight, preterm infants: the role of negative emotionality in the specification of effects. Developmental Psychopathology, 14(2), 311-32.

Blum, N. J., Taubman, B., Tretina, L., \& Heyward, Y. (2002). Maternal ratings of infant intensity and 
distractibility: Relationship with crying duration in the second month of life. Archives of Pediatrics Adolescent Medicine, 156(3), 286-290.

Bradley, R. H., Whiteside, L., Caldwell, B. M., Casey, P. H., Kelleher, K. J., Pope, S. K., Swanson, M., Barret, K., \& Cross, D. (1993). Maternal IQ, the home environment, and child IQ in low birthweight, premature children. International Journal of Behavioral Development, 16, 61-74.

Buss, A. H., \& Plomin, R. (1984). Temperament: Early developing personality traits. Hillsdale, NJ: Lawrence Erlbaum Associates.

Cicchetti, D., \& Cohen, D. J. (1995). Perspectives on developmental psychology. In D. Cicchetti, \& D. J Cohen (Eds.), Developmental Psychology: Theory and methods (pp. 3-22). New York: John Wiley \& Sons.

Damman, O., Walther, H., Allers, B., Schröder, M., Drescher, J., Lutz, D., Veelken, N., \& Schulte, F.J. (1996). Development of a regional cohort of very low-birthweight children at six years: Cognitive abilities are associated with neurological disability and social background. Developmental Medicine \& Child Neurology, 38, 97-108.

Foulder-Hughes, L. A., \& Cooke, RW.I. (2003). Motor, cognitive, and behavioural disorders in children born very preterm. Developmental Medicine \& Child Neurology, 45, 97-103.

Garber, J. (1984). Classification of childhood psychopathology: A developmental perspective. Child Development, 55(1), 30-48.

Goldsmith, H. H. (1983). Genetic influences on personality from infancy to adulthood. Child Development, 54(2), 331-55.

Goldsmith, H. H. (1994). Studying temperament via construction of the Toddler Behavior Questionnaire. Child Development, 67(1), 218-235.

Gray, R. F., Indurkhya, A., \& McCormick, M. (2004). Prevalence, stability and predictors of clinically significant behavior problems in low birth weight children at 3,5 , and 8 years of age. Pediatrics, 114(3), 736-743.
Grunau, R. E., Holsti, L., Haley, D. W., Oberlander, T., Weinberg, J., Solimano, A., Whitfield, M. F., Fitzgerald, C., \& Yu, W. (2005). Neonatal procedural pain exposure predicts lower cortisol and behavioral reactivity in preterm infants in the NICU. Pain, 113(3), 293-300.

Grunau, R. E., Oberlander, T. F, Whitfield, M. F., Fitzgerald, C., Morison, S. J., \& Saul, J. P. (2001). Pain reactivity in former extremely low birth weight infants at corrected age 8 months compared with term born controls. Infant Behavior and Development, 24(1), 44-55.

Grunau, R. E., Weinberg, J., \& Whitfield, M. F. (2004). Neonatal procedural pain and preterm infant cortisol response to novelty at 8 months. Pediatrics, 114 (1), 77-84.

Grunau, R. E., Whitfield, M. F., \& Petrie, J. (1994). Pain sensitivity and temperament in extremely low-birth-weight premature toddlers and preterm and full-term controls. Pain, 58(3), 341-346.

Gutman, L. M., Sameroff, A. J., \& Cole, R. (2003). Academic growth curve trajectories from $1^{\text {st }}$ grade to $12^{\text {th }}$ grade: Effects of multiple social risk factors and preschool child factors. Developmental Psychology, 39 (4), 777-790.

Helgadóttir, H. L., \& Wilson, M. E. (2004). Temperament and pain in 3 to 7-year-old children undergoing tonsillectomy. Journal of Pediatric Nursing, 19 (3), 204-213.

Holmbeck, G. N. (2002). Post-hoc probing of significant moderational and mediational effects in studies of pediatric population. Journal of Pediatric Psychology, 27 (1), 87-96.

Honjo, S., Mizuno, R., Sechiyama, H., Sasaki, Y., Kaneko, H., Nishide, T., Nagata, M., Sobajima, H., Nagai, Y., Ando, T., \& Nishide, Y. (2002). Temperament of low birth weight infants and childrearing stress: Comparison with full-term healthy infants. Early Child Development and Care, 172 (1), 65-75.

Kerestes, G. (2005). Maternal ratings of temperamental characteristics of healthy premature infants are indistinguishable from those of full-term infants. Croatian Medical Journal, $46(1), 36-44$. 
Kopp, C. B., \& Kaler, S.R. (1989). Risk in infancy origins and implications. American Psychologist, 44 (2), 224-230.

Larroque, B., N'guyen, The Tich, S., Guedeney, A., Marchand, L., \& Burguet, A. (2005). Temperament at 9 months of very preterm infants born at less than 29 weeks' gestation: the Epipage study. Journal of Developmental and Behavioral Pediatrics, 26 (1), 48-55.

Laucht, M., Esser, G., Schmidt, M. H. (1997). Developmental outcomes of infants born with biological and psychological risks. Journal of Child Psychology and Psychiatry, 38 (7), 843-853.

Laucht, M., Esser, G., \& Schmidt, M. H. (2002). Vulnerability and resilience in the development of children at risk: The role of early mother-child interaction. Revista de Psiquiatria Clínica, 29 (1), 20-27.

Laucht, M., Esser, G., \& Schmidt, M. H. (2001). Differential development of infants at risk for psychopathology: the moderating role of early maternal responsivity. Developmental Medicine \& Child Neurology, 45(5), 292-300.

Linhares, M. B. M., Bordin, M. B. M., \& Carvalho, A. E. V. (2004). Aspectos do desenvolvimento psicológico da criança ex-prematura na fase escolar. In E. M. Marturano, M. B. M. Linhares \& S. R. Loureiro (Orgs.), Vulnerabilidade e proteção: Indicadores na trajetória de desenvolvimento do escolar (pp. 75-1061). São Paulo: Casa do Psicólogo.

Luthar, S. S., Burack, J. A, Cicchetti, D., \& Weisz, J. R. (1997). Developmental Psychopatology: Perspectives on adjustment, risk, and disorder (pp. 15-21). New York: Cambridge University Press.

Morison, S. J., Holsti, L., Grunau, R. E., Whitfield, M. F., Oberlander, T. F., Chan, H. W., \& Williams, L. (2003). Are there developmentally distinct motor indicators of pain in preterm infants? Early Human Development, 72 (2), 131-146.

Muris, P., \& Ollendick, T. H. (2005). The role of temperament in the etiology of child psychopathology. Clinical Child and Family Psychology Review, 8 (4), 271-289.
Putnam, S. P., Ellis, L. K., \& Rothbart, M. K. (2001). The structure of temperament from infancy through adolescence. In A. Eliasz \& A. Angleitner (Eds.), Advances in research on temperament (pp. 165182). Germany: Pabst Scientific.

Rocha, E. M., Prkachin, K. M., Beaumont, S. L., Hardy, C. L., \& Zumbo, B. D. (2003). Pain reactivity and somatization in kindergarten-age children. Journal of Pediatric Psychology, 28 (1), 47-57.

Rothbart, M. K. (1981). Measurement of temperament in infancy. Child Development, 52(2), 569-578.

Rothbart, M. K. (1989). Biological processes of temperament. In G. Kohnstamm, J. Bates \& M.K. Rothbart (Eds.), Temperament in childhood (pp.77-110). Chichester, England: Wiley.

Rothbart, M. K., Posner, M. I., \& Hershey, K. L. (1995). Temperament, attention, and developmental psychopathology. In D. Cicchetti $\&$ D. J. Cohen (Eds.), Manual of developmental psychopathology: Theory and methods (pp.315355). New York: John Wiley and Sons.

Rothbart, M. K., \& Putnam, S (2002). Temperament and socialization. In L. Pulkinnem \& A. Caspi (Eds.). Paths to successful development: Personality in the life course (pp. 19-45). Cambridge: Cambridge University Press.

Rothbart, M. K., Ellis, L. K., Rueda, M. R., \& Posner, M. I. (2003). Developing mechanisms of temperamental effortful control. Journal of Personality, 71 (6), 1113-1143.

Rothbart, M. K. (2004). Commentary: Differentiated measures of temperament and multiple pathways to childhood disorders. Journal of Clinical Child and Adolescence Psychology, 33(1), 82-87.

Rutter, M. (1987). Psychological resilience and protective mechanisms. American Journal of Orthopsychiatry, 57(3), 316-331.

Rutter, M. (1994). Temperament: Changing concepts and implications. In W. B. Carey \& S. C. McDevitt (Eds.), Prevention and early intervention: individual differences as risk factors for the mental health of children. A festchrift for Stella Chess and Alexander Thomas (pp. 23-34). New York: Brunner/ Mazel. 
Rutter, M., \& Sroufe, L. A. (2000). Developmental psychopathology: Concepts and challenges. Development and Psychopathology,12, 265- 296.

Sajaniemi, N., Salokorpi, T., \& Von Wendt L. (1998). Temperament profiles and their role in neurodevelopmental assessed preterm children at two years of age. European Child \& Adolescent Psychiatry, 7(3), 145-152.

Sameroff, A. J. (2000). Developmental systems and psychopathology. Developmental and Psychopathology, 12, 297-312.

Saylor, C. F., Boyce, G. C., \& Price, C. (2003). Early predictors of school-age behavior problems and social skills in children with intraventricular hemorrhage (IVH) and/or extremely low birthweight (ELBW). Child Psychiatry and Human Development, 33(3), 175-192.

Schothorst, P. F., \& Van Engeland, H. (1996). Longterm behavioral sequelae of prematurity. Journal of the American Academy of Child and Adolescent Psychiatry, 32 (2), 175-183.

Strelau, J., \& Eliaz, A (1994). Temperament risk factors for type A behavior pattern in adolescents. In W. B. Carey \& S. C. McDevitt (Eds.), Prevention and early intervention: Individual differences as risk factors for the mental health of children. A Festchrift for Stella Chess and Alexander Thomas (pp. 36-41). New York: Brunner/ Mazel.

Taylor, H. G., Hack, M., Klein, N., \& Schatschneider, C. (1995). Achievement and children with birth weight less than $750 \mathrm{~g}$ with normal cognitive abilities: Evidence for specific learning disabilities. Journal of Pediatric Psychology, 20 (6), 703-719.

Thomas, A., \& Chess, S. (1977). Temperament and Development. New York: Brunner/ Mazel.

Van Bakel, H. J.A., \& Riksen-Walraven, J. M. (2004). Stress reactivity in 15-month-old infants: Links with infant temperament, cognitive competence, and attachment security. Developmental Psychobiology, 44(3), 157-167.

Wasserman, R. C., Diblasio, C. M., Bond, L. A., Young, P. C., \& Colletti, R. B. (1990). Infant temperament and school age behavior: 6-year longitudinal study in a pediatric practice. Pediatrics, 85 (5), 801-807.

Weiss, S. J., St. John-Seed, M., \& Wilson, P. (2004). The temperament of pre-term, low birth weight infants and its potential biological substrates. Research in Nursing \& Health, 27(6), 392-402.

Werner, E. E. (1986). A longitudinal study of perinatal risk. In D. C. Farran \& J. D. McKinney (Eds.), Risk in intellectual and psychosocial development (pp. 61-68). Orlando: Academic Press.

Whitfield, M. F., \& Grunau, R. E. (2000). Behavior, pain perception, and the extremely low-birth weight survivor. Clinics and Perinatology, 27 (2), 363- 379.

Yunes, M. A. (2003). Psicologia positiva e resiliência: O foco no indivíduo e na família. Psicologia em Estudo, 8(especial), 75-84.

Yunes, M. A., \& Szymanski, H. (2001). Resiliência: Noção, conceitos afins e considerações críticas. In J. Tavares (Orgs.), Resiliência e educação (pp. 13-42). São Paulo: Cortez.

Zimmerman, L. K., \& Stansburry, K. (2004). The influence of emotion regulation, level of shyness, and habituation on the neuroendocrine response of three-year-old children. Psychoneuroendocrinology, 29, 973-982.

Endereço para correspondência:

Vivian Caroline Klein, Laboratório de Pesquisa em Prevenção em Problemas de Desenvolvimento e Comportamento da Criança, FMRP-USP - Prédio da Saúde Mental. Rua Tenente Catão Roxo, 2650. CEP: 14048-900, Ribeirão Preto - SP, Brasil. E-mail: kleinvivian@hotmail.com ou linhares@fmrp.usp.br

Artigo recebido em 22/09/2006.

Aceito para publicação em 15/03/2007.

As autoras contam com o apoio da Fundação de Amparo à Pesquisa do Estado de São Paulo (FAPESP), Coordenação de Aperfeiçoamento de Pessoal de Nível Superior (CAPES) e Conselho Nacional de Desenvolvimento Científico e Tecnológico (CNPq). 